\title{
Cost-effectiveness of the implementation of an enhanced recovery protocol for colorectal surgery
}

\author{
D. Roulin ${ }^{1}$, A. Donadini ${ }^{1}$, S. Gander ${ }^{2}$, A.-C. Griesser ${ }^{3}$, C. Blanc ${ }^{2}$, M. Hübner ${ }^{1}$, M. Schäfer ${ }^{1}$ \\ and N. Demartines ${ }^{1}$ \\ Departments of ${ }^{1}$ Visceral Surgery and ${ }^{2}$ Anaesthesiology, and ${ }^{3}$ Medical Direction, University Hospital of Lausanne, Lausanne, Switzerland \\ Correspondence to: Professor N. Demartines, Department of Visceral Surgery, Centre Hospitalier Universitaire Vaudois, CH-1011 Lausanne, Switzerland \\ (e-mail: demartines@chuv.ch)
}

\begin{abstract}
Background: Enhanced recovery protocols may reduce postoperative complications and length of hospital stay. However, the implementation of these protocols requires time and financial investment. This study evaluated the cost-effectiveness of enhanced recovery implementation.

Methods: The first 50 consecutive patients treated during implementation of an enhanced recovery programme were compared with $\mathbf{5 0}$ consecutive patients treated in the year before its introduction. The enhanced recovery protocol principally implemented preoperative counselling, reduced preoperative fasting, preoperative carbohydrate loading, avoidance of premedication, optimized fluid balance, standardized postoperative analgesia, use of a no-drain policy, as well as early nutrition and mobilization. Length of stay, readmissions and complications within 30 days were compared. A cost-minimization analysis was performed.

Results: Hospital stay was significantly shorter in the enhanced recovery group: median 7 (interquartile range 5-12) versus 10 (7-18) days $(P=0.003)$; two patients were readmitted in each group. The rate of severe complications was lower in the enhanced recovery group (12 versus 20 per cent), but there was no difference in overall morbidity. The mean saving per patient in the enhanced recovery group was $€ 1651$. Conclusion: Enhanced recovery is cost-effective, with savings evident even in the initial implementation period.
\end{abstract}

Presented to the 99th Annual Congress of the Swiss Surgical Society, Davos, Switzerland, June 2012, and the Seventh Annual Meeting of the European Society of Coloproctology, Vienna, Austria, September 2012; published in abstract form as Swiss Knife 2012; 9: 21 and Colorectal Dis 2012; 14: 39

Paper accepted 24 April 2013

Published online in Wiley Online Library (www.bjs.co.uk). DOI: 10.1002/bjs.9184

\section{Introduction}

Enhanced recovery is a multimodal perioperative approach aiming to attenuate the patient's stress response in order to achieve low complication rates and hence a more rapid postoperative recovery ${ }^{1}$. Based on the available evidence, standardized clinical pathways have been developed covering all areas of a patient's journey through the surgical process ${ }^{2,3}$. The current enhanced recovery protocol for colorectal surgery includes more than 20 interventions, ranging from reduced preoperative fasting to an optimized fluid balance and use of a no-drain policy ${ }^{4}$. It has been demonstrated that postoperative complications may be reduced, with faster postoperative recovery ${ }^{5-8}$. Despite these encouraging results, uptake of perioperative enhanced recovery programmes in colorectal surgery has been slow in Europe and the $\mathrm{USA}^{9-12}$. This might be related to the fact that successful clinical implementation requires considerable effort to assemble an interdisciplinary team, as well as some financial investment ${ }^{3}$.

Previous studies have not detailed costs of implementation of the protocol ${ }^{13-15}$, or included mostly open colonic surgery ${ }^{16}$. The present study aimed to assess the costeffectiveness of implementation of an enhanced recovery programme with a defined protocol for laparoscopic and open colorectal surgery.

\section{Methods}

An enhanced recovery protocol for colorectal surgery was developed and implemented at the university hospital of 
Table 1 Perioperative care elements for colorectal surgery

\begin{tabular}{|c|c|c|}
\hline & Enhanced recovery & Standard care \\
\hline Patient counselling ${ }^{\star}$ & Preadmission counselling + written information & No standardized information \\
\hline Fasting & Clear fluids until $2 \mathrm{~h}$, solids $6 \mathrm{~h}$ before surgery & Solid and fluid fasting from midnight \\
\hline Carbohydrate drinks ${ }^{\star}$ & $800 \mathrm{ml}$ on evening and $400 \mathrm{ml} 2 \mathrm{~h}$ before surgery & No carbohydrate drinks \\
\hline Premedication ${ }^{\star}$ & No premedication & Premedication with oral midazolam $7.5 \mathrm{mg}$ \\
\hline Thromboprophylaxis* & LMW heparin $12 \mathrm{~h}$ before surgery, IPC & LMW heparin $12 \mathrm{~h}$ before surgery \\
\hline PONV prophylaxis* & PONV prophylaxis $\dagger$ & No PONV prophylaxis \\
\hline Balanced intravenous fluids* & $\begin{array}{l}\text { Intraoperative crystalloids } 500-1000 \mathrm{ml} \text { for surgery } \\
<3 \mathrm{~h} \text {; otherwise crystalloids } 1500 \mathrm{ml} \text { and colloids } \\
500-1000 \mathrm{ml} \text {. Postoperative crystalloids } 500 \mathrm{ml} \\
\text { during the first } 24 \mathrm{~h} \text {, then stop }\end{array}$ & No guidance \\
\hline Postoperative analgesia & $\begin{array}{l}\text { Thoracic epidural analgesia for laparotomy. Epidural or } \\
\text { PCA for laparoscopy } \neq . \text { Paracetamol, ibuprofen and } \\
\text { oxycodone-naloxone only for breakthrough pain }\end{array}$ & No standardized postoperative analgesia \\
\hline Abdominal drains & No routine abdominal drainage $\S$ & Abdominal drainage at surgeon's discretion \\
\hline Bladder catheter ${ }^{\star}$ & Removal on postoperative day 1 & Removal at surgeon's discretion \\
\hline Nutrition ${ }^{\star}$ & $\begin{array}{l}\text { Free fluid } 4 \mathrm{~h} \text { after surgery. Normal diet from day of } \\
\text { surgery. Two nutritional supplements per day }\end{array}$ & $\begin{array}{l}\text { No standardized reintroduction of fluid and } \\
\text { food }\end{array}$ \\
\hline Systematic laxatives* & Oral magnesium hydroxide & No systematic laxatives \\
\hline Mobilization* & $\begin{array}{l}\text { Out of bed at least } 15 \mathrm{~min} \text { on the day of surgery; at } \\
\text { least } 6 \mathrm{~h} \text { per day thereafter }\end{array}$ & No mobilization objectives \\
\hline Systematic audit & Systematic audit; bimonthly meeting & No audit \\
\hline Bowel preparation* & \multicolumn{2}{|c|}{ Avoidance of bowel preparation } \\
\hline Antibiotic prophylaxis* & \multicolumn{2}{|c|}{ Cefuroxime $1.5 \mathrm{~g}+$ metronidazole $500 \mathrm{mgIl}$} \\
\hline Hypothermia prevention* & \multicolumn{2}{|c|}{ Active warming (air blanket) } \\
\hline Nasogastric tubes* & \multicolumn{2}{|c|}{ No routine postoperative gastric tube } \\
\hline
\end{tabular}

*Items used to calculate mean compliance rate. $\dagger$ Droperidol $1 \mathrm{mg}$ at induction; ondansetron $4 \mathrm{mg}$ with or without betamethasone $4 \mathrm{mg}$ at the end of operation; betamethasone only for women or non-smokers or those with previous history of postoperative nausea and vomiting (PONV). $\ddagger$ Removal after $48 \mathrm{~h}$ for colonic and $72 \mathrm{~h}$ for rectal surgery. §Except for rectal resection below peritoneal reflection. II30 min before incision. LMW, low molecular weight; IPC, intermittent pneumatic compression; PCA, patient-controlled analgesia.

Lausanne, a tertiary referral centre for colorectal surgery. A local interdisciplinary team of surgeons, anaesthetists, nutritionists, nurses and administrators underwent the formal training provided by the ERAS ${ }^{\circledR}$ Society. All protocols, including patient information and clinical pathways, as well as the database, the ERAS ${ }^{\circledR}$ Interactive Audit System, were provided by the ERAS ${ }^{\circledR}$ Society. From February 2011, the local team attended the first seminars and adapted the protocols provided to the local needs of the university hospital of Lausanne. In June 2011, the first patients subjected to the enhanced recovery pathway were included, and the implementation period ended in November 2011. During the first 2 months, only elective colonic resections were included in order to allow familiarization with this new protocol. Afterwards, all patients scheduled for elective laparoscopic or open colonic or rectal operations were included. Exclusion criteria were: age below 18 years and emergency operations within $24 \mathrm{~h}$ after admission.

Consecutive patients treated during implementation of the enhanced recovery programme were compared with those treated in the year before its introduction. Patients were identified from a hospital database. Inclusion and exclusion criteria were similar for both groups. Informed consent was obtained from all patients before surgery. The study was approved by the institutional review board.

The protocol is outlined on Table 1. Of note, the following discharge criteria in the enhanced recovery group were used: sufficient postoperative pain control with oral analgesia, tolerance of solid food, passage of flatus or faeces, and independent ambulation. A dedicated study nurse contacted all patients 5 days after discharge from hospital, and a clinical assessment was performed in the outpatient department after 30 days. Patients in the control group received standard postoperative care and were discharged from hospital at the surgeon's discretion.

\section{Assessment of postoperative morbidity and mortality}

Postoperative complications were graded according to severity on a validated five-point scale ${ }^{17}$. Grades IIIa to $\mathrm{IVb}$ were defined as severe complications. More than one complication per patient was possible, but only the highest ranked complication was used for final analysis. Postoperative mortality (grade V) was defined as death during the first 30 days after surgery or during the hospital stay after the index operation. 


\section{Cost-minimization analysis}

The cost-minimization analysis was performed from a healthcare provider's perspective to assess savings per patient in hospital. Costs were obtained in Swiss francs $(\mathrm{CHF})$, and then converted to euros $(€)$. The exchange rate used was $€ 1=1.20 \mathrm{CHF}$, which was the official rate on 22 June 2012.

\section{Implementation costs}

The protocol implementation costs included variable costs (patient's logbook, carbohydrate drinks and nutritional supplements) and fixed costs (enhanced recovery implementation costs, travel and lunch expenses of multidisciplinary team training, and cost of full-time dedicated nurse recruited in June 2011).

Direct in-hospital costs included all costs that could be attributed to any resource consumption during the hospital stay for the index operation and, if applicable, a readmission stay. For each patient, inpatient costs were those incurred from the day of admission until the day of discharge, with cost allocations provided by the university hospital accounting database. Anaesthesia costs were calculated per minute based on the duration of anaesthesia (including costs of anaesthetists, nurse anaesthetists, materials and drugs). Operating room costs were calculated per minute based on duration of occupation. Surgeon's costs were included under medical care. Intensive and intermediate care unit costs were calculated per day based on the Project Research in Nursing (PRN) score ${ }^{18}$. Medical care costs included all clinical activities performed by doctors, including surgeons, but not costs incurred by anaesthetists, radiologists and pathologists. Nursing care costs were those incurred per day outside intensive and intermediate care units, based on the PRN score. Costs of housing were accounted per day and administration costs were accounted per patient admission.

\section{Sensitivity analysis}

When fixed costs, which are independent of the number of patients, are allocated on an individual level, they show large variation in relation to the number of patients treated within the study period. Therefore, a sensitivity analysis of the costs of enhanced recovery implementation was carried out. The number of patients treated within the study period was varied ( \pm 50 per cent $)$ in order to assess its impact on the cost-minimization results.

\section{Statistical analysis}

Continuous variables were compared using two-tailed Mann-Whitney or Student's $t$ test, as appropriate. Categorical variables were analysed by means of Fisher's exact test or $\chi^{2}$ test. Despite an imbalance in the distribution of cost data, the arithmetic mean was considered as the

Table 2 Baseline characteristics of patients undergoing colorectal surgery

\begin{tabular}{|c|c|c|c|}
\hline & Enhanced recovery $(n=50)$ & Standard care $(n=50)$ & $P \dagger$ \\
\hline Age (years) ${ }^{\star}$ & $65.0(17.9)$ & $65 \cdot 0(13 \cdot 6)$ & 0.995 \\
\hline Sex ratio $(M: F)$ & $28: 22$ & $25: 25$ & $0.689 \ddagger$ \\
\hline Body mass index $\left(\mathrm{kg} / \mathrm{m}^{2}\right)^{\star}$ & $26 \cdot 7(5 \cdot 0)$ & $27 \cdot 3(4 \cdot 1)$ & 0.357 \\
\hline ASA grade & & & $0.265 \ddagger$ \\
\hline I-II & 39 & 33 & \\
\hline III & 11 & 17 & \\
\hline \multicolumn{4}{|l|}{ P-POSSUM ${ }^{\star}$} \\
\hline Physiological & $18(6)$ & 19(4) & 0.341 \\
\hline Operative & $13(4)$ & $13(4)$ & 0.557 \\
\hline Surgical approach & & & $0.016 \%$ \\
\hline Laparoscopic & 33 & 20 & \\
\hline Open & 17 & 30 & \\
\hline Type of operation & & & $0.528 \S$ \\
\hline Colectomy & 27 & 33 & \\
\hline Anterior resection of rectum & 14 & 9 & \\
\hline Abdominoperineal resection & 2 & 3 & \\
\hline Other & 7 & 5 & \\
\hline Diagnosis & & & $0.294 \S$ \\
\hline Adenocarcinoma & 36 & 29 & \\
\hline Diverticular disease & 6 & 11 & \\
\hline Other & 8 & 10 & \\
\hline
\end{tabular}

*Values are mean(s.d.). ASA, American Society of Anesthesiologists; P-POSSUM, Portsmouth modification of the Physiological and Operative Severity Score for the enUmeration of Mortality and morbidity ${ }^{20}$. $†$ Unpaired $t$ test, except $\ddagger$ Fisher’s exact test and $\S \chi^{2}$ test. 
Table 3 Clinical outcomes of patients undergoing colorectal surgery

\begin{tabular}{|c|c|c|c|}
\hline & Enhanced recovery $(n=50)$ & Standard care $(n=50)$ & $P \dagger$ \\
\hline Mean compliance with enhanced recovery protocol (\%) & 71 & 36 & \\
\hline Time to first passage of flatus (days) ${ }^{\star}$ & $2(1-2)$ & $3(2-4)$ & 0.005 \\
\hline Time to first passage of stool (days) ${ }^{*}$ & $3(2-4)$ & $4(3-5)$ & $<0.001$ \\
\hline \multicolumn{4}{|l|}{ Patients with a complication within 30 days } \\
\hline Grade I-II & 24 & 19 & $0.414+$ \\
\hline Grade IIIa-IVb & 6 & 10 & $0.287 \ddagger$ \\
\hline Postoperative stay (days)* & $7(5-12)$ & $10(7-18)$ & 0.003 \\
\hline
\end{tabular}

*Values are median (interquartile range). $†$ Mann-Whitney $U$ test, except $\ddagger$ Fisher's exact test.

Table 4 Total individual costs of primary hospital stay

\begin{tabular}{|c|c|c|c|c|}
\hline & \multicolumn{2}{|c|}{ Mean cost per patient $(€)^{\star}$} & \multirow[b]{2}{*}{ Mean difference $(€) \dagger$} & \multirow[b]{2}{*}{$P \ddagger$} \\
\hline & Enhanced recovery $(n=50)$ & Standard care $(n=50)$ & & \\
\hline Total intraoperative costs & $10573(9563,11667)$ & $8801(7822,10083)$ & $1772(-5,3633)$ & 0.031 \\
\hline Disposable materials & $2494(2183,2808)$ & $1639(1368,1951)$ & $855(409,1258)$ & 0.002 \\
\hline Anaesthesia and operating room & $8079(7144,9262)$ & $7162(6336,8263)$ & $917(-651,2600)$ & 0.212 \\
\hline Total preoperative + postoperative costs & $13735(11453,16448)$ & $18169(14$ 058, 23 118) & $-4434(-1028,644)$ & 0.102 \\
\hline Intensive and intermediate care & $2045(1367,2936)$ & $3077(1864,4566)$ & $-1032(-2803,-420)$ & 0.249 \\
\hline Medical care & $3122(2650,3721)$ & $3653(3083,4338)$ & $-531(-1406,372)$ & 0.224 \\
\hline Nursing care & $4368(3409,5663)$ & $5538(4129,7458)$ & $-1170(-3449,1255)$ & 0.304 \\
\hline Physiotherapy & $194(135,271)$ & $338(228,468)$ & $-144(-286,-14)$ & 0.058 \\
\hline Medication & $588(455,760)$ & $966(663,1313)$ & $-378(-713,-76)$ & 0.048 \\
\hline Blood transfusion and testing & $261(163,373)$ & $393(223,583)$ & $-132(-373,93)$ & 0.261 \\
\hline Laboratory & $476(367,592)$ & $993(718,1334)$ & $-517(-845,-238)$ & 0.006 \\
\hline Radiology & $143(77,214)$ & $422(265,611)$ & $-279(-475,-93)$ & 0.012 \\
\hline Housing and administration & $2538(2219,2893)$ & $2789(2358,3357)$ & $-251(-891,-388)$ & 0.429 \\
\hline
\end{tabular}

*Values are means, derived from 1000 non-parametric bootstrap replicates, with 95 per cent confidence intervals. $\dagger$ Enhanced recovery minus standard care. $\$$ Bootstrapped $t$ test.

most informative measure from the decision-makers' perspective. The non-parametric bootstrap test, which was described for pharmacoeconomic cost analysis with moderate sample sizes ${ }^{19}$, was used for the cost analysis in the present study. $P<0.050$ was considered statistically significant. All analyses were performed using SPSS $^{\circledR} 20$ (IBM, Armonk, New York, USA).

\section{Results}

The first 50 consecutive patients treated during implementation of an enhanced recovery programme were compared with 50 consecutive patients treated in the year before its introduction. The two groups were similar regarding age, sex, body mass index, American Society of Anesthesiologists physical status grade, Portsmouth modification of the Physiological and Operative Severity Score for the enUmeration of Mortality and morbidity (P-POSSUM $)^{20}$, diagnosis and type of operation (Table 2). Of note, there were significantly more laparoscopic operations in the enhanced recovery group $(P=0 \cdot 016)$. The number of conversions from laparoscopy to open surgery was comparable: six and eight patients in the enhanced recovery and standard groups respectively $(P=0 \cdot 774)$. The mean(s.d.) duration of operation was 261(101) $\mathrm{min}$ in the enhanced recovery group and 238(98) $\mathrm{min}$ in the standard group $(P$ $=0.248)$.

\section{Postoperative outcomes}

The mean rate of adherence to the protocol, defined as the numbers of elements fulfilled divided by the total number of items (Table 1), was 71 per cent in the enhanced recovery group compared with 36 per cent in the standard group. Adherence to each perioperative care element is detailed in Table S1 (supporting information).

Postoperative recovery was significantly faster among patients treated within the enhanced recovery protocol (Table 3). Overall, there were 48 complications in the enhanced recovery group and 60 in the standard group, but the difference did not reach statistical significance $(P=$ $0 \cdot 640$ ). Only one patient in the standard group died within 30 days of surgery (mortality rate 2 per cent). Postoperative stay was significantly shorter in the enhanced recovery group, with a reduction in median stay of 3 days $(P=0.003)$. There were two readmissions within 30 postoperative days 
Table 5 Summary of cost-minimization analysis

\begin{tabular}{lrr} 
& \multicolumn{2}{c}{ Mean cost per patient $(€)$} \\
\cline { 2 - 3 } & Enhanced recovery $(n=50)$ & Standard care $(n=50)$ \\
Enhanced recovery implementation & 1011 & 0 \\
Intraoperative cost & 10573 & 8801 \\
Preoperative + postoperative costs & 13735 & 18169 \\
Total costs & 25319 & 1772 \\
-4434 & 26970
\end{tabular}

*Enhanced recovery minus standard care.

in each group. The total readmission length of stay was 13 days in the enhanced recovery group and 36 days in the standard group.

\section{Cost-minimization analysis}

The variable costs for carbohydrate drinks and nutritional supplements for each patient in the enhanced recovery group were $€ 75$, and that for the patient's logbook was $€ 4$. Fixed costs for implementation of the enhanced recovery programme and travel and lunch expenses of the multidisciplinary team were $€ 15000$ and $€ 4108$ respectively. A full-time study nurse was recruited at $€ 27500$ for the 6 months during which the first 50 patients were recruited to the enhanced recovery programme. By distributing the fixed costs among these 50 patients and adding the individual variable costs, $€ 1011$ was attributed to each patient.

Operative costs per patient were significantly higher in the enhanced recovery group, with a difference of $€ 1772$ ( $P=0.031)$. More disposable materials, such as staplers, trocars and dissection instruments, were used during laparoscopy. As a higher proportion of patients in the enhanced recovery group underwent laparoscopic colorectal surgery, overall costs of disposable materials were increased. Furthermore, laparoscopic surgery took slightly longer than open surgery. However, there was an overall cost saving of $€ 4434$ per patient in the enhanced recovery group regarding total preoperative and postoperative costs (Table 4). Accounting for all the different costs, a mean overall saving of $€ 1651$ per patient was observed in favour of the enhanced recovery group (Table 5).

\section{Sensitivity analysis}

As most of the enhanced recovery implementation costs were fixed, the mean costs per patient were closely related to the number of patients treated in the study period. By varying the number of patients by \pm 50 per cent, the mean implementation costs were $€ 1943$ per patient for 25 patients and $€ 700$ per patient for 75 patients. The total cost balance remained in favour of the enhanced recovery group, estimated at $€ 718$ and $€ 1961$ for 25 and 75 patients respectively.

\section{Discussion}

This study found that implementation of an enhanced recovery programme for colorectal surgery in a Swiss university hospital led to cost savings. Standardization of perioperative patient management and a significantly shortened hospital stay contributed to this economic benefit. Commonly raised criticisms of cost analysis studies are the lack of complete cost data, which restricts conclusions, and that only overall economic effects can be shown; savings or losses per patient are rarely depicted. The present study included all direct costs related to perioperative management as well as all costs of the protocol, including its implementation, charged to the number of included patients.

The significant reduction in length of stay might explain the cost reduction observed, as has been shown previously after implementation of a clinical pathway ${ }^{21}$. However, the length of stay has a limited impact on overall costs because hospital days are not equivalent economically. The first few postoperative days are generally more costly than those just before discharge ${ }^{22}$. Moreover, postoperative complications have a major impact on hospital costs, and complications and their severity are the strongest indicator of hospital charges $^{23}$. In the present study, there was only a small difference in the overall number of complications between groups, but complications in the enhanced recovery group were less severe.

Available data on cost-minimization of enhanced recovery protocols in colorectal surgery are sparse. A previous study, which did not include implementation costs, reported a comparable decrease of $€ 1782$ following the implementation of a clinical pathway for elective open colonic surgery ${ }^{24}$. Complication rates were similar before and after introduction of the clinical pathway, and readmission rates increased from 2 to 10 per cent 
with the accelerated pathway. A recent randomized clinical trial in China reported a statistically significant total cost reduction of $€ 263$ per patient in an enhanced recovery programme ${ }^{14}$. However, there was no detailed costminimization analysis and the development costs were not considered. The only study on colonic surgery to include costs of implementation of such a programme was performed in New Zealand; an overall cost saving of approximately $€ 4521$ was reported ${ }^{16}$. In contrast to the present study, only 10 per cent of all interventions were carried out laparoscopically. The LAparoscopy and/or FAst track multimodal management versus standard care (LAFA) study found laparoscopy in combination with a dedicated enhanced recovery programme to be the optimal perioperative approach, with similar in-hospital costs in the four groups ${ }^{15}$.

The present study has some limitations. First, retrospective data collection for the standard group could have led to incomplete identification of postoperative events. Despite the higher rate of laparoscopic operation associated with higher intraoperative costs in the enhanced recovery group, implementation of the programme was still cost-effective. Thus, if the rate of laparoscopy had been similar in both groups, the cost reduction in the enhanced recovery group would have been even greater ${ }^{25}$. Finally, as the enhanced recovery protocol resulted in reduced length of stay, there may have been a transfer of costs from the hospital to the community. These costs, including loss of productivity, were not evaluated in the present study.

\section{Acknowledgements}

The authors acknowledge the support provided by all members of the enhanced recovery team in Lausanne, including the Nutrition Unit led by P. Coti Bertrand, as well as charge nurses, A. Jannot and L. Cudre, and the senior ward manager, A. Cotillard. Special thanks go to V. Addor, the enhanced recovery-dedicated clinical nurse. Disclosure: The authors declare no conflict of interest.

\section{References}

1 Kehlet H, Wilmore DW. Multimodal strategies to improve surgical outcome. Am 7 Surg 2002; 183: 630-641.

2 Gustafsson UO, Hausel J, Thorell A, Ljungqvist O, Soop M, Nygren J. Adherence to the enhanced recovery after surgery protocol and outcomes after colorectal cancer surgery. Arch Surg 2011; 146: 571-577.

3 Maessen J, Dejong CH, Hausel J, Nygren J, Lassen K, Andersen $\mathrm{J}$ et al. A protocol is not enough to implement an enhanced recovery programme for colorectal resection. $\mathrm{Br} \mathrm{F}$ Surg 2007; 94: 224-231.
4 Lassen K, Soop M, Nygren J, Cox PB, Hendry PO, Spies C et al.; Enhanced Recovery After Surgery (ERAS) Group. Consensus review of optimal perioperative care in colorectal surgery: Enhanced Recovery After Surgery (ERAS) Group recommendations. Arch Surg 2009; 144: 961-969.

5 Varadhan KK, Neal KR, Dejong CH, Fearon KC, Ljungqvist $\mathrm{O}$, Lobo DN. The enhanced recovery after surgery (ERAS) pathway for patients undergoing major elective open colorectal surgery: a meta-analysis of randomized controlled trials. Clin Nutr 2010; 29: 434-440.

6 Spanjersberg WR, Reurings J, Keus F, van Laarhoven CJ. Fast track surgery versus conventional recovery strategies for colorectal surgery. Cochrane Database Syst Rev 2011; (2)CD007635.

7 Adamina M, Kehlet H, Tomlinson GA, Senagore AJ, Delaney CP. Enhanced recovery pathways optimize health outcomes and resource utilization: a meta-analysis of randomized controlled trials in colorectal surgery. Surgery 2011; 149: 830-840.

8 Muller S, Zalunardo MP, Hubner M, Clavien PA, Demartines N; Zurich Fast Track Study Group. A fast-track program reduces complications and length of hospital stay after open colonic surgery. Gastroenterology 2009; 136: 842-847.

9 Lassen K, Hannemann P, Ljungqvist O, Fearon K, Dejong $\mathrm{CH}$, von Meyenfeldt MF et al. Enhanced Recovery After Surgery Group. Patterns in current perioperative practice: survey of colorectal surgeons in five northern European countries. BMF 2005; 330: 1420-1421.

10 Kehlet H, Buchler MW, Beart RW Jr, Billingham RP, Williamson R. Care after colonic operation - is it evidence-based? Results from a multinational survey in Europe and the United States. 7 Am Coll Surg 2006; 202: $45-54$.

11 Hasenberg T, Keese M, Langle F, Reibenwein B, Schindler $\mathrm{K}$, Herold A et al. 'Fast-track' colonic surgery in Austria and Germany - results from the survey on patterns in current perioperative practice. Colorectal Dis 2009; 11: 162-167.

12 Arsalani-Zadeh R, Ullah S, Khan S, Macfie J. Current pattern of perioperative practice in elective colorectal surgery; a questionnaire survey of ACPGBI members. Int 7 Surg 2010; 8: 294-298.

13 King PM, Blazeby JM, Ewings P, Longman RJ, Kipling RM, Franks PJ et al. The influence of an enhanced recovery programme on clinical outcomes, costs and quality of life after surgery for colorectal cancer. Colorectal Dis 2006; 8: 506-513.

14 Ren L, Zhu D, Wei Y, Pan X, Liang L, Xu J et al. Enhanced Recovery After Surgery (ERAS) program attenuates stress and accelerates recovery in patients after radical resection for colorectal cancer: a prospective randomized controlled trial. World 7 Surg 2012; 36: 407-414.

15 Vlug MS, Wind J, Hollmann MW, Ubbink DT, Cense HA, Engel AF et al.; LAFA study group. Laparoscopy in combination with fast track multimodal management is the best perioperative strategy in patients undergoing colonic 
surgery: a randomized clinical trial (LAFA-study). Ann Surg 2011; 254: 868-875.

16 Sammour T, Zargar-Shoshtari K, Bhat A, Kahokehr A, Hill AG. A programme of Enhanced Recovery After Surgery (ERAS) is a cost-effective intervention in elective colonic surgery. N Z Med 7 2010; 123: 61-70.

17 Dindo D, Demartines N, Clavien PA. Classification of surgical complications: a new proposal with evaluation in a cohort of 6336 patients and results of a survey. Ann Surg 2004; 240: 205-213.

18 Chagnon M, Audette LM, Lebrum L, Tilquin C. A patient classification system by level of nursing care requirements. Nurs Res 1978; 27: 107-112.

19 Thompson SG, Barber JA. How should cost data in pragmatic randomised trials be analysed? BMF 2000; 320: 1197-1200.

20 Prytherch DR, Whiteley MS, Higgins B, Weaver PC, Prout WG, Powell SJ. POSSUM and Portsmouth POSSUM for predicting mortality. Physiological and Operative Severity Score for the enUmeration of Mortality and morbidity. $\operatorname{Br} 7$ Surg 1998; 85: 1217-1220.
21 Pritts TA, Nussbaum MS, Flesch LV, Fegelman EJ, Parikh AA, Fischer JE. Implementation of a clinical pathway decreases length of stay and cost for bowel resection. Ann Surg 1999; 230: 728-733.

22 Taheri PA, Butz DA, Greenfield LJ. Length of stay has minimal impact on the cost of hospital admission. $7 \mathrm{Am}$ Coll Surg 2000; 191: 123-130.

23 Vonlanthen R, Slankamenac K, Breitenstein S, Puhan MA, Muller MK, Hahnloser D et al. The impact of complications on costs of major surgical procedures: a cost analysis of 1200 patients. Ann Surg 2011; 254: 907-913.

24 Stephen AE, Berger DL. Shortened length of stay and hospital cost reduction with implementation of an accelerated clinical care pathway after elective colon resection. Surgery 2003; 133: 277-282.

25 King PM, Blazeby JM, Ewings P, Franks PJ, Longman RJ, Kendrick AH et al. Randomized clinical trial comparing laparoscopic and open surgery for colorectal cancer within an enhanced recovery programme. Br 7 Surg 2006; 93: 300-308.

\section{Supporting information}

Additional supporting information may be found in the online version of this article:

Table S1 Adherence to each perioperative care element (Word document) 